\title{
Contexto sociocultural y aprendizaje escolar ${ }^{1}$
}

\section{Socio-cultural context and school learning}

José Eduardo Cifuentes Garzón ${ }^{2}$

\section{Resumen}

El presente artículo tiene como objetivo reflexionar sobre la incidencia del contexto sociocultural en los procesos de enseñanza y de aprendizaje, a fin de ser tenido en cuenta en el diseño, ejecución y evaluación de las experiencias didácticas. Por eso resalta el deber ser del maestro frente al papel del contexto en los procesos de aprendizaje, la relación estrecha entre aprendizaje, contexto y desarrollo y el impacto de los factores socioculturales en el desempeño escolar. El autor destaca la necesidad de abrir espacios en las diferentes instituciones educativas, con el propósito de caracterizar a la población estudiantil,; con la intención de recoger información para entender su realidad y diseñar algunas estrategias encaminadas a mitigar los efectos de las condiciones socioculturales que interfieren en los procesos de construcción del conocimiento. El éxito de la tarea educativa, está en gran parte en la manera como el docente articula los contextos y el desarrollo de los estudiantes al diseño y ejecución de la práctica pedagógica.

Palabras clave: Contexto sociocultural; Desarrollo; Aprendizaje; Práctica educativa; Experiencias didácticas.

\begin{abstract}
This article aims to reflect on the impact of the sociocultural context on teaching and learning processes, in order to take it into account in the design, implementation and evaluation of didactic experiences. For this reason, this paper highlights the teacher's ought to be against the role of context in learning processes, the close relationship between learning, context, and development, and the impact of sociocultural factors on school performance. The author emphasizes the need to open spaces in the different educational institutions with the purpose of characterizing the student population, with the intention of collecting information for understanding their reality and designing some strategies aim at mitigating the effects of sociocultural conditions that interfere in the process of knowledge construction. The success of the educational task is largely in the way the teacher articulates the contexts and development of students in the design and implementation of pedagogical practice.
\end{abstract}

Keywords: Sociocultural context; Development; Learning; Educational practice.

\footnotetext{
Artículo del curso: "Contextos de desarrollo y aprendizaje" de la Maestría en Pedagogía de La Universidad de La Sabana (2014) y de reflexiones abordadas en seminarios en el marco del Doctorado en Educación y Sociedad de La Universidad de La Salle (2015-2016).

${ }^{2}$ Magister en Pedagogía de la Universidad de La Sabana. Docente de Ciencias Sociales en la Institución Educativa Departamental Minipí de Quijano de La Palma Cundinamarca.

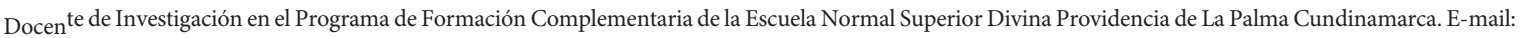
josecifuentes1980@gmail.com
}

Cómo citar este artículo: Cifuentes, G. J. (2017). Contexto sociocultural y aprendizaje escolar. Revista Hojas y Hablas, (14), 107-122. doi: 10.29151/hojasyhablas.n14a8 


\section{Introducción}

...Es a través de la pedagogía como se logra la mediación fundamental entre la cultura y el estudiante para promover el desarrollo infantil $\mathrm{y}$ adolescente, para permitir que avance en sus procesos de pensamiento y lenguaje... de su comprensión del funcionamiento del mundo y, además, que vayan abriendo su perspectiva para comprender las relaciones físicas y sociales que ayudan a explicar el mundo en que viven y la estructura de su cultura... (Isaza, 2012, p. 25)

La escuela en el marco de la tarea de educar, debe procurar el desarrollo de diversas habilidades en los estudiantes, según las particularidades de cada uno. El maestro es quien puede identificar las potencialidades y limitaciones de sus discípulos, desde las interacciones en el aula y el despliegue de las demás acciones propias de los Proyectos Educativos Institucionales. En este sentido, el maestro actúa como un escultor, encargado de moldear o refinar las dimensiones cognitivas, procedimentales y actitudinales de los estudiantes, como se ilustra a continuación:

Un niño vecino de un gran taller de escultura, entró un día en el estudio del escultor y vio en él un gigantesco bloque de piedra. Dos meses después, al regresar el niño, encontró en su lugar una preciosa estatua ecuestre. Y volviéndose al escultor, le preguntó: “¿cómo sabías tú que dentro de aquel bloque había un caballo?”

La frase del pequeño era bastante más que una gracia infantil. Porque de verdad el caballo estaba, en realidad, ya dentro de aquel bloque. La capacidad artística del escultor consistió precisamente en eso: en saber ver el caballo que había dentro e irle quitando al bloque de piedra todo cuanto le sobraba. El escultor no trabajó añadiendo trozos de caballo al bloque de piedra, sino liberando la piedra de todo aquello que impedía mostrar el caballo ideal encriptado en su interior. El artista supo "ver" dentro, eso que nadie veía. Ese fue su arte.

Pienso todo esto al comprender como con la educación de los humanos pasa algo muy parecido. La palabra educar viene del latín educare (sacar de dentro). La verdadera genialidad del educador no consiste en añadirle al niño las cosas faltantes, sino descubrir los tesoros de cada estudiante y saberlos sacar a la luz. (Adaptado de José L. Martín Descalzo)

La anterior reflexión invita a pensar en la misión de los maestros en relación con el descubrimiento de las potencialidades de los niños, niñas y jóvenes en la escuela. Sin embargo, en la mayoría de los casos, el maestro se preocupa más por añadir al estudiante los conocimientos faltantes, los cuales él cree tener, al desconocer los niveles de desarrollo y los diversos contextos de los estudiantes que inciden notablemente en sus procesos de aprendizaje. Por tal razón, el presente artículo quiere una reflexión en torno al contexto sociocultural y su relación con los aprendizajes adquiridos en la escuela, esto genera una brecha entre lo planeado por las instituciones educativas y los aprendizajes reales de los estudiantes. Sumado a esto, el sistema de evaluación procura medir los niveles de aprendizajes, centrado en la mayoría en pruebas escritas, que no demuestran del todo, las comprensiones y el desarrollo de competencias. En consecuencia, resulta importante repensar las maneras de planear, ejecutar y evaluar el acto educativo para poder transformar las prácticas de aula en desempeños destinados a descubrir y refinar talentos.

De esta manera, el artículo se organiza en cuatro partes: la primera hace referencia a una mirada 
al contexto desde algunos resultados de las pruebas saber, la segunda al deber ser del docente frente al papel del contexto en los procesos de aprendizaje, la tercera a la triada inseparable aprendizaje, contexto sociocultural y desarrollo, y finalmente en la cuarta se presenta unas reflexiones acerca de la importancia de analizar el contexto como punto de partida para lograr transformaciones en las prácticas educativas.

\section{Una mirada al contexto desde los resultados de las pruebas saber}

El Instituto Colombiano para la Evaluación de la Educación -ICFES- con la intención de "identificar las variables que más influencian el rendimiento escolar de los estudiantes (...) ha aplicado una serie de cuestionarios que recogen información sobre el contexto de los estudiantes, sus familias y la institución educativa"(...) (2016, p. 5). En este sentido, para analizar el impacto de los factores asociados al rendimiento académico, optó por el modelo teórico "Contexto, Insumos, Procesos y Productos, CIPP":

En la categoría de contexto encontramos los factores que influyen en el sistema educativo (...) incluye aquellas variables externas a la institución educativa que moldean los resultados académicos. Dentro del contexto son relevantes las características sociales, económicas y culturales de los estudiantes y sus familias, lo cual implica un amplio rango de variables cuya definición depende de la situación social de cada contexto nacional y local. La categoría de insumos (...) está enfocada principalmente en los recursos de la institución educativa; sin embargo, esta categoría también considera el historial educativo de los estudiantes como un factor importante al valorar el aprendi- zaje. La categoría de procesos (...) incluye las actividades regulares desarrolladas en el colegio y dentro del salón de clases para cumplir con los objetivos de enseñanza en los establecimientos escolares. Finalmente, los productos representan los resultados esperados de las actividades realizadas en la institución educativa y en el salón de clases. (Icfes, 2016, p. 11-12)

En el presente artículo de reflexión, se profundizará en la primera categoría del CIPP. "El contexto en el que se desarrolla el aprendizaje (y que depende de las condiciones sociales, económicas y culturales de cada país) representa la dimensión del marco de factores asociados que más influye en el aprendizaje (Unesco, 2015b; Unesco, 2010, citados por el Icfes, 2016, p. 17)". En la tabla 1, se presenta las variables y aspectos de esta categoría:

Tabla 1. Variables del contexto

\begin{tabular}{|c|c|c|}
\hline Categoría & Variables & Aspectos \\
\hline \multirow[t]{2}{*}{ Contexto } & $\begin{array}{c}\text { Características } \\
\text { de los estudiantes }\end{array}$ & $\begin{array}{l}\text { Género. Edad. } \\
\text { Trabajo infantil. Nivel } \\
\text { socioeconómico familiar. } \\
\text { Violencia en el entorno } \\
\text { del hogar. Involucramiento } \\
\text { parental. Pertenencia a un } \\
\text { pueblo originario o etnia. } \\
\text { Necesidades educativas } \\
\text { especiales. Autoconcepto } \\
\text { académico. Motivación. } \\
\text { Estrategias de aprendizaje. }\end{array}$ \\
\hline & $\begin{array}{l}\text { Características } \\
\text { de las escuelas }\end{array}$ & $\begin{array}{l}\text { Zona en la que se ubica la } \\
\text { institución educativa (urbana } \\
\text { a rural). Dependencia } \\
\text { administrativa. Tamaño de } \\
\text { la institución educativa y } \\
\text { el aula de clases. Nivel } \\
\text { socioeconómico promedio } \\
\text { por aula y establecimiento. } \\
\text { Violencia en el entorno de la } \\
\text { institución educativa. }\end{array}$ \\
\hline
\end{tabular}

Fuente: Icfes (2016) 
De acuerdo con el Icfes, (2016):

Las características sociodemográficas, económicas y culturales de los estudiantes y sus familias han sido consideradas como variables importantes en la explicación de los resultados escolares desde la publicación del Informe Coleman (Coleman, 1966), lo cual ha sido confirmado por diversos estudios internacionales posteriores (OECD, 2011a, 2011b; Unesco, 2015b; Unesco, 2010). (Icfes, 2016, p. 18)

[En relación a las características de las escuelas] la literatura muestra brechas en los resultados académicos de distintos tipos de instituciones educativas, diferenciadas por el nivel socioeconómico, promedio de la población que atienden (Reardon, 2011), su ubicación geográfica (Unesco, 2015a) y, en menor medida, el entorno que las rodea (Sanbonmatsu, Kling, Duncan \& Brooks-Gunn, 2006). (Icfes, 2016, p. 23).

El Icfes, recoge la información del contexto a partir de un cuestionario sociodemográfico, a fin de calcular el nivel socioeconómico (NSE) para cada estudiante. Esta medición tiene en cuenta los siguientes aspectos:

Tabla 2: Perfil de los hogares de los estudiantes según nivel socioeconómico

\begin{tabular}{|c|c|}
\hline NSE & Perfil \\
\hline 1 & $\begin{array}{l}\text { - Los padres o acudientes tienen educación básica primaria; en algunos casos no han recibido ningún tipo de } \\
\text { - } \quad \text { Los macación. } \\
\text { - } \quad \text { De manera general, la vivienda posee inodoro con conexión a pozo séptico, pero en algunos casos está conectado } \\
\text { - } \text { al alcantarillado. } \\
\text { - } \quad \text { Ln el hogar viven entre cinco y siete personas. } \\
\text { - Las personas que habitan en la vivienda disponen de uno a dos cuartos para dormir. }\end{array}$ \\
\hline 2 & $\begin{array}{l}\text { - Los padres o acudientes tienen educación primaria o secundaria. } \\
\text { - Los materiales de los pisos del hogar son cemento o gravilla y, en varios casos, baldosa, tableta, ladrillo o vinilo. } \\
\text { - En inodoro está conectado al alcantarillado. } \\
\text { - La vivienda tiene entre dos y cuatro cuartos o piezas. } \\
\text { - Las personas que habitan en la vivienda disponen de dos a tres cuartos para dormir. }\end{array}$ \\
\hline 3 & $\begin{array}{l}\text { - Los padres o acudientes tienen educación secundaria. } \\
\text { - } \quad \text { Los materiales de los pisos del hogar son baldosa, tableta, ladrillo o vinilo. } \\
\text { - } \quad \text { En el hogar viven entre tres y cinco personas. } \\
\text { - La vivienda tiene entre tres y cuatro cuartos o piezas. Las personas que habitan en la vivienda disponen de dos a tres } \\
\text { cuartos para dormir. }\end{array}$ \\
\hline 4 & $\begin{array}{l}\text { - Los padres o acudientes tienen educación universitaria y posgrado; muy pocos tienen solo educación secundaria. } \\
\text { - Los materiales de los pisos del hogar son baldosa, tableta, ladrillo o vinilo. } \\
\text { - El inodoro está conectado al alcantarillado. } \\
\text { - En el hogar viven entre tres y cuatro personas. } \\
\text { - La vivienda tiene cuatro o más cuartos o piezas. } \\
\text { - Las personas que habitan en la vivienda disponen de tres a cinco cuartos para dormir. }\end{array}$ \\
\hline
\end{tabular}

Fuente: Icfes (2017, p. 90). 
Teniendo en cuenta lo anterior, las instituciones educativas son clasificadas en cuatro niveles: NSE 1, NSE 2, NSE 3 y NSE 4, según el promedio del índice de sus estudiantes. La tabla 3 presenta los porcentajes de establecimientos por nivel socioeconómico en los últimos años:

Tabla 3: Porcentajes de establecimientos por nivel socioeconómico

\begin{tabular}{|c|c|c|c|c|c|}
\hline Año & NSE 1 & NSE 2 & NSE 3 & NSE 4 & Indefinido \\
\hline 2009 & $40,3 \%$ & $13,8 \%$ & $11,4 \%$ & $22,9 \%$ & $11,6 \%$ \\
\hline 2012 & $26,4 \%$ & $13,7 \%$ & $11,1 \%$ & $16,0 \%$ & $32,8 \%$ \\
\hline 2013 & $24,0 \%$ & $12,4 \%$ & $11,0 \%$ & $20,9 \%$ & $31,8 \%$ \\
\hline 2014 & $24,9 \%$ & $12,9 \%$ & $11,3 \%$ & $19,9 \%$ & $31,0 \%$ \\
\hline 2015 & $26,2 \%$ & $14,3 \%$ & $13,0 \%$ & $24,9 \%$ & $21,5 \%$ \\
\hline 2016 & $26,1 \%$ & $35,6 \%$ & $25,7 \%$ & $10,2 \%$ & $2,4 \%$ \\
\hline
\end{tabular}

Fuente: Icfes (2017, p. 12).
Para ilustrar la manera como afecta el nivel socioeconómico en los aprendizajes de los estudiantes, las gráficas siguientes muestran los resultados nacionales de la prueba Saber $9^{\circ}$ de los años 2009, 2012-2016, en las áreas de lenguaje, matemáticas, ciencias naturales y pensamiento ciudadano, según indican las barras en su respectivo orden. Dado que la aplicación de las pruebas de ciencias naturales y pensamiento ciudadano es alternada año a año en noveno, no existe información para el área de ciencias naturales en 2013 y 2015 y para pensamiento ciudadano en 2014 y 2016.

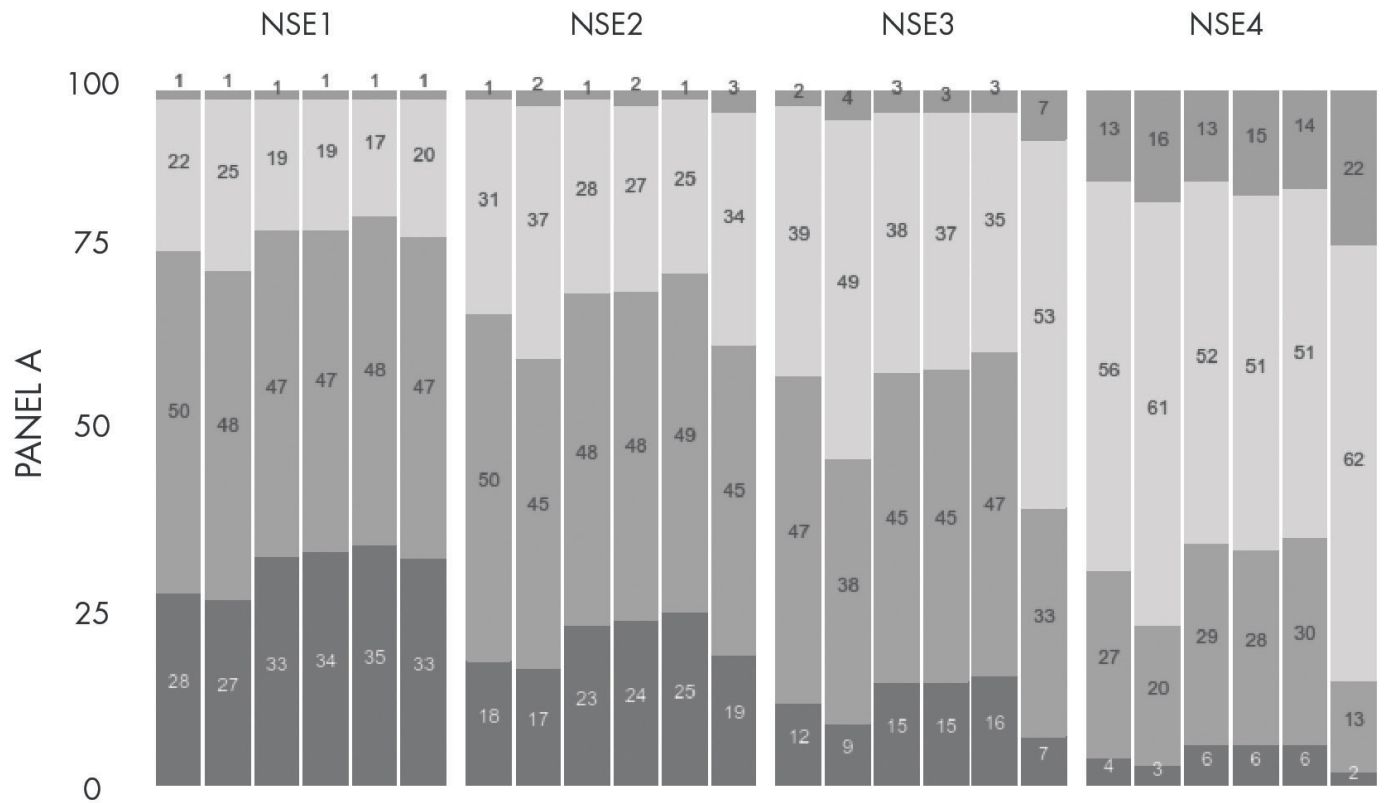

Gráfica 1. Resultados nacionales por nivel socioeconómico en Saber $9^{\circ}$, área de lenguaje Fuente: Icfes, 2017, p. 57. 

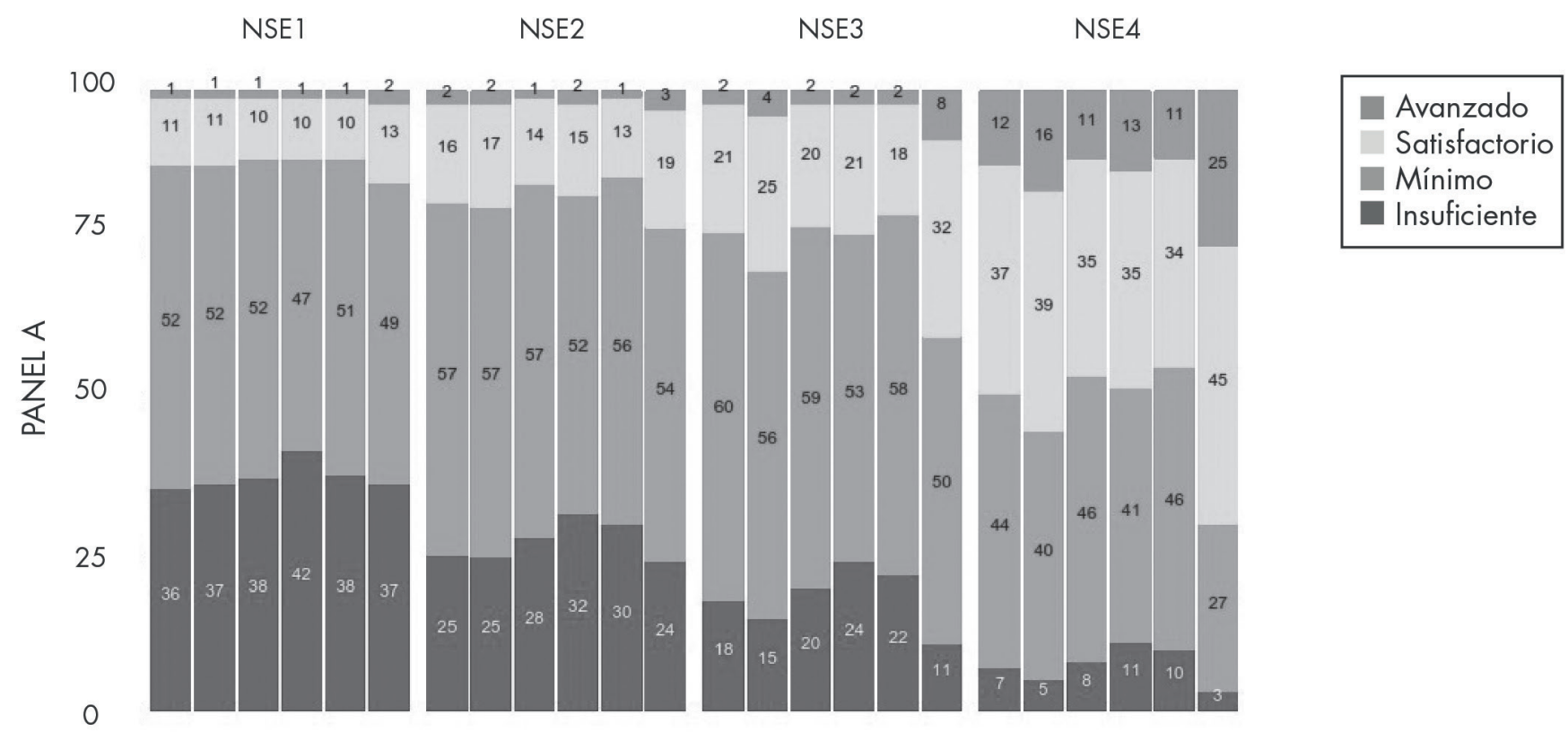

Gráfica 2. Resultados nacionales por nivel socioeconómico en Saber $9^{\circ}$, área de matemáticas Fuente: Icfes $(2017$, p. 61$)$.

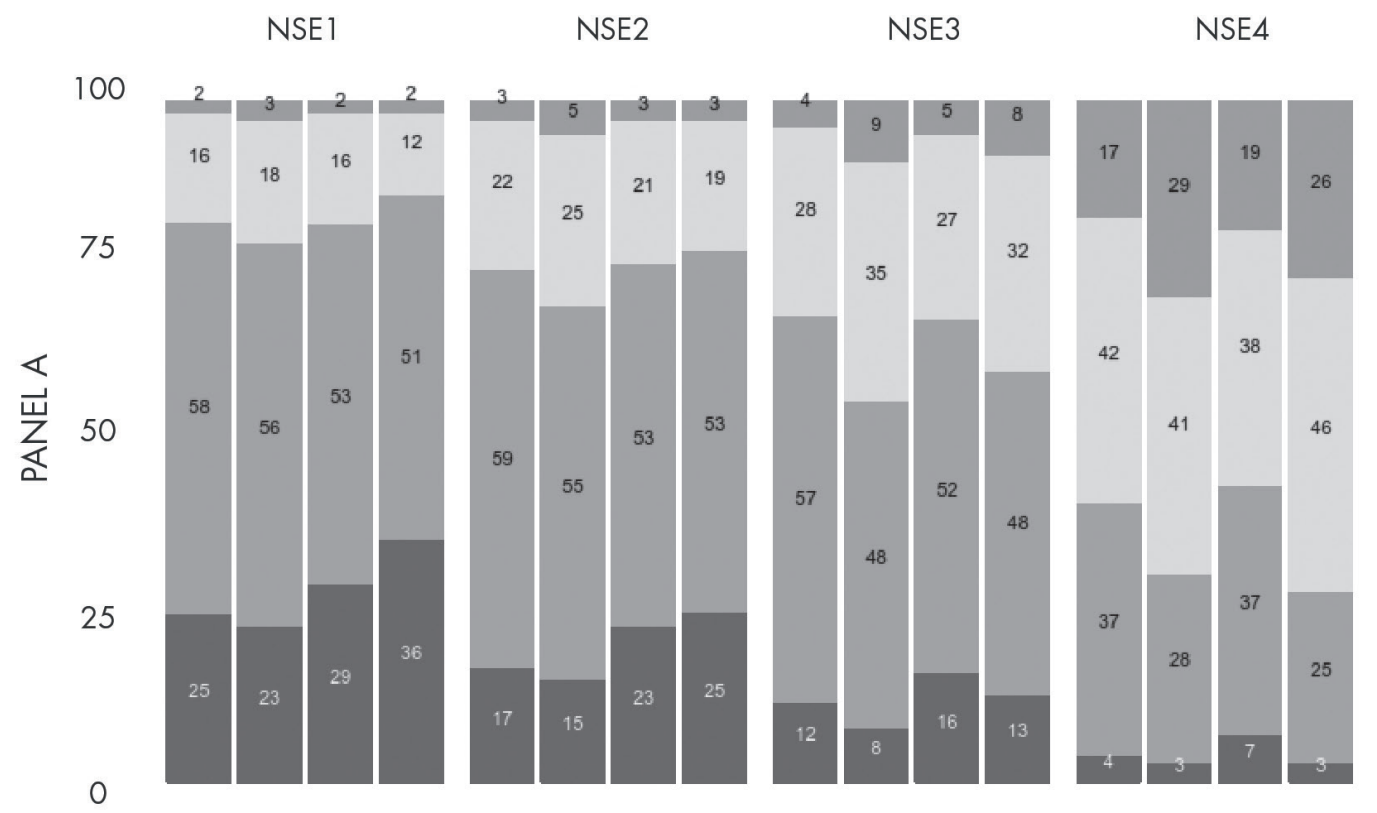

Gráfica 3. Resultados nacionales por nivel socioeconómico en Saber $9^{\circ}$, área de ciencias naturales Fuente: Icfes $(2017$, p. 64). 


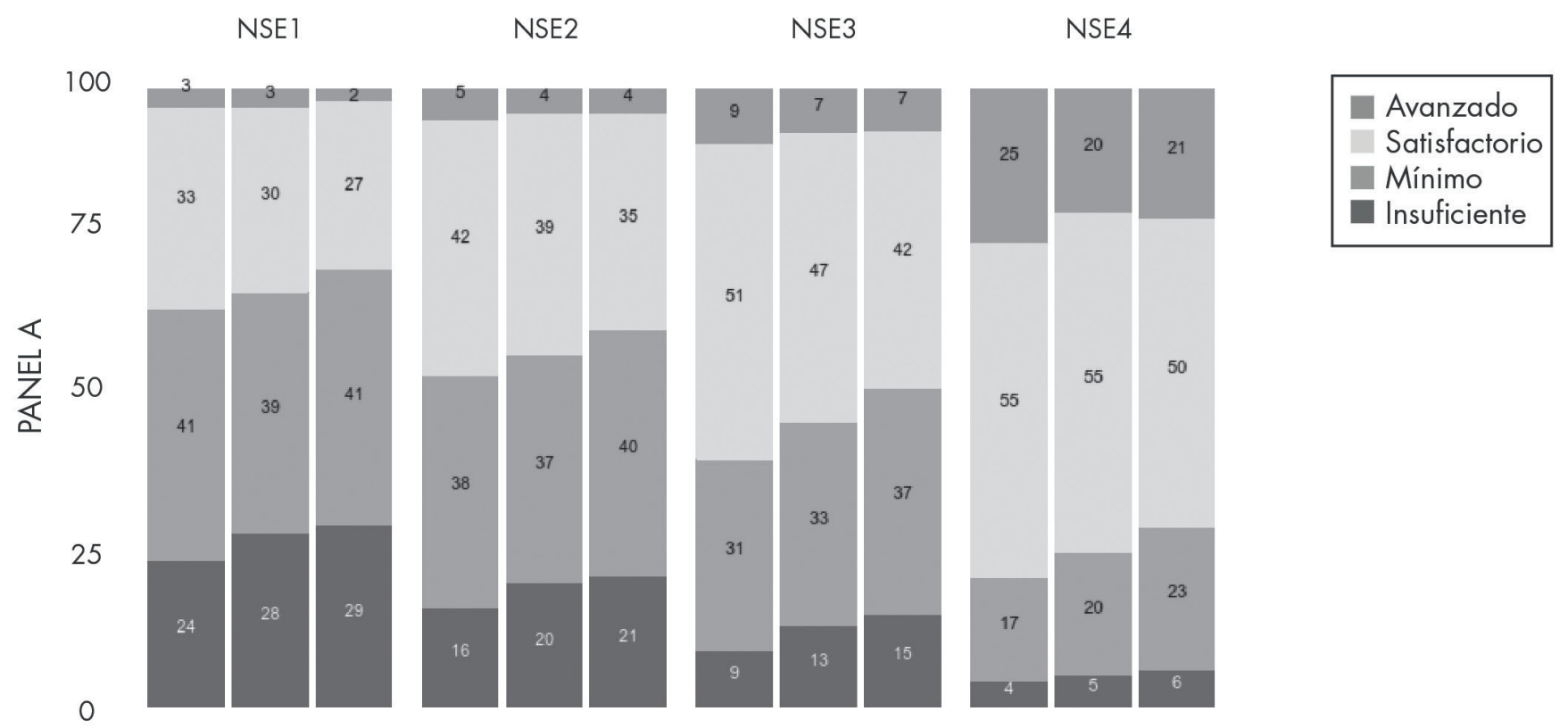

Gráfica 4. Resultados nacionales por nivel socioeconómico en Saber $9^{\circ}$, área de pensamiento ciudadano Fuente: Icfes (2017, p. 67).

De acuerdo a lo anterior, en las áreas de matemáticas, lenguaje, ciencias naturales y pensamiento ciudadano, se evidencia que el puntaje promedio obtenido por los establecimientos educativos es considerablemente mayor a medida que aumenta el nivel socioeconómico. Se mantiene la tendencia de un alto porcentaje de estudiantes con nivel socioeconómico 1 y 2 en insuficiente, mientras que en los de nivel socioeconómico 4 , se amplían los porcentajes en avanzado y disminuye el insuficiente. Es decir:

Para que los niños puedan ir a la escuela y participar exitosamente de las clases es necesario que estén adecuadamente alimentados y sanos, que vivan en un medio que no les signifique obstáculos a las prácticas educativas, y que hayan internalizado un conjunto de representaciones, valores y actitudes que los dispongan favorablemente para el aprendizaje escolar. (López y Tedesco, 2002, p. 10)

\section{El deber ser del docente frente al papel del contexto en los procesos de aprendizaje}

En los últimos años, en el sector educativo colombiano, se ha observado una particular inquietud respecto al impacto del contexto en el desarrollo y aprendizajes de las niñas, niños y jóvenes; al ser este un tema central en la discusión permanente entre los diferentes actores educativos interesados en el mejoramiento de los procesos de enseñanza y de aprendizaje. El interés por estudiar el contexto y la relación con el aprendizaje, obedece a la necesidad urgente de propiciar ambientes adecuados, con presencia efectiva de normas y acuerdos, relaciones interpersonales adecuadas y prácticas de enseñanza docente pertinentes (Icfes, 2016), para ofrecer una educación de calidad, centrada en los aprendizajes significativos de los estudiantes, encaminada a mejorar sus condiciones de vida y el progreso de la misma sociedad.

Muestra de lo anterior, es el interés del Ministerio de Educación Nacional (2012), en destacar 
el papel del contexto en el aprendizaje, al incluir en la evaluación de competencias pedagógicas para ascenso y reubicación salarial de los maestros, competencias orientadas a las problemáticas asociadas con los procesos de enseñanza y aprendizaje, entre ellas: la articulación de contextos y la integración del desarrollo del estudiante a la práctica educativa.

En cuanto a la competencia para la articulación de contextos del estudiante a la práctica educativa, el Ministerio de Educación, evaluó si el docente incorporaba las experiencias y los saberes de los estudiantes de sus escenarios cotidianos y sus comunidades de origen a los procesos de enseñanza y aprendizaje de la escuela, con el fin de lograr un diálogo con sentido entre ellos y si creaba además, las condiciones pedagógicas para lograr aprendizajes situados, al integrar los contextos escolares (institucionales) y extraescolares (socioculturales) de los estudiantes.

En relación con la competencia para la integración del desarrollo de habilidades y aprendizajes en el estudiante y la relación con la práctica educativa, se quería fortalecer el saber y el saber ser del maestro, al tener en cuenta si el maestro desarrollaba procesos de mediano y largo plazo destinados a integrar los procesos de enseñanza y aprendizaje con las dimensiones de desarrollo psicológico y físico de los estudiantes. Evaluaba si el maestro realizaba ajustes y reformulaciones de fondo en la dimensión social, cognitiva, espiritual y comunicativa en la vida escolar, desde las interacciones en el aula, en las que se propician a futuro, mecanismos de convivencia y resolución de conflictos y relaciones con los padres de familia, a fin de comprender en profundidad los procesos de desarrollo de aprendizaje y habilidades en los estudiantes. Además, pretendía que el maestro orientara su acción educativa de forma individualizada, con el propósito de dar cuenta, entre otros aspectos, de los estilos cognitivos de cada estudiante, de sus formas de regular y expresar sus emociones $y$ afectos, y de las características del ambiente familiar y comunitario, los cuales inciden en su desarrollo psicológico. (MEN, 2012).

Con las nuevas formas de evaluar a los maestros para ascender o reubicación salarial (vídeo de las prácticas de aula, autoevaluación, encuestas a estudiantes...), el MEN (2015), estableció aspectos a evaluar a los docentes de aula como parte de la Evaluación de Carácter Diagnóstico Formativa (ECDF). En relación a la práctica y el contexto escolar, se destaca el siguiente criterio, componente y aspectos a evaluar:

Tabla 4: Criterios de la Evaluación de Carácter Diagnóstico Formativa

\begin{tabular}{|c|c|c|}
\hline Criterio & Componente & Aspecto a evaluar \\
\hline $\begin{array}{l}\text { Contexto de } \\
\text { la práctica } \\
\text { educativa y } \\
\text { pedagógica } \\
\text { del docente }\end{array}$ & $\begin{array}{c}\text { Contexto social } \\
\text { económico } \\
\text { y cultural }\end{array}$ & $\begin{array}{l}\text { - El maestro demuestra } \\
\text { comprensión y apropiación } \\
\text { de las especi-aficidades de } \\
\text { su contexto, sus posibilidades } \\
\text { y limitantes. } \\
\text { - La práctica del maestro } \\
\text { muestra flexibilidad con } \\
\text { respecto a los aspectos } \\
\text { fundamentales del entorno } \\
\text { y las necesidades de sus } \\
\text { estudiantes. } \\
\text { El maestro diseña estrategias } \\
\text { para tratar de vincular a las } \\
\text { familias en el proceso de } \\
\text { formación de los estudiantes. }\end{array}$ \\
\hline
\end{tabular}

Fuente: MEN (2015).

En este mismo sentido, los Lineamientos Curriculares de Matemáticas (1998), expedidos por el Ministerio de Educación Nacional, señalan la relación del contexto con los ambientes que rodean al estudiante y le dan sentido al aprendizaje. Cuestiones como las condiciones sociales y culturales tanto locales como internacionales, el tipo de interacciones, los intereses, las creencias, así como las condiciones económicas del grupo social en donde se concreta el acto educativo, deben tenerse 
en cuenta en el diseño y ejecución de experiencias didácticas. Esto implica, acudir a la didáctica y preguntarse:

... ¿cómo aprenden los niños? ¿Cómo enseñar el contenido - conceptos, nociones, elementos- de una disciplina?, y ¿Cómo generar una adecuada apropiación del contenido de las disciplinas en términos de saber?... y del lado del profesor la pregunta clave seguirá siendo ¿Qué enseña un profesor y cuál es su relación con el conocimiento de su disciplina? (Zambrano, 2016, p. 57)

Sin embargo, existen serios problemas en hacer realidad el corpus de la pedagogía y la didáctica en las aulas de clase. Al respecto, Zuluaga (2007), señala:

La escuela está perdiendo el monopolio enciclopédico del saber... Asisten a este proceso los medios de comunicación y la informática. Ellos y otros factores sociales y políticos hacen que el lugar de la pedagogía y la didáctica, la escuela y el aula, estén dejando de ser el lugar de producción de discurso pedagógico y didáctico... (p. 117)

De acuerdo a lo anterior, el maestro como investigador debe reflexionar sobre sus prácticas, analizar los diversos factores asociados a los procesos de enseñanza, entre ellos el contexto, a fin de direccionar estrategias de didácticas que faciliten el aprendizaje en los estudiantes.

Continuando con la idea de las didácticas del maestro y su relación con los resultados en los aprendizajes, para el Icfes (2014), factores como la formación, experiencia y las percepciones de los docentes inciden en el desempeño de los niños, niñas y jóvenes. Frente al nivel de formación y experiencia, según el Icfes (2014), en Colombia la formación de los docentes es heterogénea y en el sector público, hay menos correspondencia entre el área de enseñanza y el área de formación del docente, sin embargo afirma no encontrarse evidencia de la relación existente entre instituciones donde la mayoría de los docentes enseñan su área de formación o tienen altos niveles de experiencia con el logro escolar de las pruebas saber.

De otra parte, el Icfes (2014), señala que mejores percepciones en cuanto a seguridad en sus métodos y prácticas de enseñanza de los maestros sobre los miembros de la comunidad educativa y de su propia institución, se relacionan positivamente con el logro escolar de los estudiantes.

En este orden de ideas, el deber ser del docente frente al papel del contexto en los procesos de aprendizaje, implica la inmersión del educador en las diversas dinámicas sociales de interacción en los estudiantes. Es decir, podría pensarse en una labor de etnógrafo educativo en perspectiva colaborativa: salir del aula junto con los estudiantes a conocer la vereda o el barrio, untarse de su comunidad, registrar los problemas y modos de vida, con la intención de construir con ellos hojas de ruta destinadas a articular los hallazgos a la planeación de clase y desarrollo de la práctica de aula.

\section{Aprendizaje, contexto sociocultural y desarrollo: triada inseparable}

El aprendizaje, el contexto y el desarrollo, juegan un papel trascendental en los procesos formativos de los diferentes niveles, en la medida en que son inherentes al acto educativo y se requiere una apropiada articulación entre estas tres dimensiones para lograr el propósito de la formación integral. El aprendizaje, según Argüelles y Nagles (2007) es entendido como un cambio en la estructura cognitiva de la persona generado por la contrastación de las nuevas representaciones, ideas y conceptos con el conocimiento previo; este cam- 
bio está influenciado por las experiencias, vivencias, sentimientos, emociones y por el contexto de interacción de cada persona. El desarrollo, según Cejudo (2006) está referido al proceso para aumentar las oportunidades de las personas, y el contexto considerado para Guzmán y Ghitis (2012) como un espacio simbólico compartido, expresado por medio del lenguaje, símbolos, costumbres, hábitos y rutinas. Esto influye directamente en las personas y contribuyen a configurar pensamientos, ideas, afectos, concepciones, representaciones sociales y culturales, al constituirse en el marco dador de sentido a las acciones y a la interpretación de las experiencias de vida.

Sin embargo, los anteriores conceptos han estado siempre circulando en el discurso de los maestros, pero distantes de las prácticas de aula, como lo afirma Jaramillo (2010)

Los tiempos de los maestros no coinciden con los tiempos de los alumnos, al parecer, por inconvenientes de lectura y/o identificación adecuada de los contextos de estos últimos. Igual suele ocurrir con el tiempo de estos y de los otros, con los tiempos de los padres de familia o de la comunidad en general. Es usual que tampoco coincidan los tiempos institucionales, con los de sus entornos. En muchas ocasiones, los tiempos de la enseñanza se han quedado rezagados con relación a los tiempos del aprendizaje. En su conjunto, la organización educativa se ha quedado atrás respecto a los nuevos contextos que impone el avasallante desarrollo social. (p.73)

Es decir, la educación se ha quedado detenida y no está acorde con los avances y cambios sociales vertiginosos de los últimos tiempos que han repercutido en la dinámica de la enseñanza y el aprendizaje. De acuerdo con Burgos y Cifuentes (2015)
Las aulas de clase, en muchas de las instituciones educativas colombianas, quedaron estancadas en el tiempo, al estar permeadas por prácticas tradicionales centradas en la adquisición de conocimientos, la rígida disciplina y la intención de moldear a los estudiantes a costumbres conservadoras, que, según el imaginario de los docentes, es la educación que necesitan, desconociendo las realidades, necesidades y aspiraciones de los niños, niñas y jóvenes... (p. 120)

Otro punto de vista, es el planteado por De Zubiría (2011), quien concibe el proceso de aprendizaje como algo incomprensible sin la mediación de la cultura sea esta dada por los padres, los maestros, los textos, la internet o la televisión. En cuanto a las tecnologías y los medios de comunicación, según Barbero (1996, p. 11), "significan para la escuela en primer lugar eso: un reto cultural, que hace visible la brecha cada día más ancha entre la cultura desde la que enseñan los maestros y aquella otra desde la que aprenden los alumnos".

Lo anterior, nos permite repensar si verdaderamente se están considerando los diferentes factores sociales, económicos y culturales en los procesos educativos. Estos aspectos afectan el aprendizaje escolar y contribuyen a nutrir los mayores problemas de los sistemas escolares contemporáneos, referidos por Torres (1995), como la falta de acceso a la escuela, la deserción y la repetición, situaciones con graves consecuencias a nivel económico y social para el progreso y desarrollo del país.

Todos los eventos ocurridos alrededor de donde se da el acto educativo interfieren notablemente para el logro o desviación de las finalidades propuestas en las instituciones educativas. Por tal razón, cobra importancia analizar con más detalle los factores socioculturales relacionados con las afectaciones en el desempeño escolar. Dichos 
factores económicos, nutricionales y parentales, son entendidos como aquellas circunstancias, medios y condiciones asumidas por los estudiantes en su cotidianidad e interfieren en el desempeño académico de las diversas áreas del saber, la convivencia social y los procesos de desarrollo del pensamiento, en términos de lo critico e investigativo, entre otros. En esta misma línea Peralta (2002), refiriéndose al estudiante y al entorno sociocultural, allí "se mueven diversos contextos: la pobreza, la violencia intrafamiliar, los estados de guerra interna y externa, el estrés de los padres, la nuclearización familiar, la globalización forzada; todo lo cual, sin duda, afecta fuertemente su vida" (p.48).

De la misma manera, el Primer Estudio Regional Comparativo y Explicativo llevado a cabo en América Latina y el Caribe en 1997 (citado por Fernández, C., Fernández, A. y Urrego, 2011) expone como el nivel socioeconómico y cultural de los estudiantes es la variable con más influencia en el aprendizaje, porque los estudiantes provenientes de familias con menos acceso a bienes materiales y culturales, y cuyos padres participan menos en la escuela, alcanzan menores niveles de logro académico. Según este estudio, el trabajo remunerado por parte de los niños incide negativamente en el aprendizaje. La participación en el mercado laboral de los niños puede ser un signo de la precariedad económica en la familia y, por otro lado, quita tiempo y energía que los niños podrían dedicar al aprendizaje.

Para el Icfes (2010, p. 26), estudios realizados en Colombia (Piñeros \& Rodríguez, 1999; Caro, 2000; WöBmann \& Fuchs, 2005; Iregui et. al., 2006), muestran la existencia de una correlación alta y significativa entre la situación económica y social de los estudiantes y su rendimiento educativo en primaria y secundaria. Es así, como las desventajas socioculturales, tales como la falta de una vivienda digna, una alimentación adecuada, los servicios en salud oportunos, la falta de opciones de recreación y acceso al arte, la cultura y el deporte y la pobreza extrema, entre otros, fomenta en los estudiantes la pérdida del deseo de superación, del gusto por el estudio y presenten dificultades en los procesos de desarrollo del pensamiento, al ser esta una realidad presentada de forma constante y evidente en los niveles actuales de deserción y repetición escolar existente en el país.

De acuerdo con el Icfes (2014), las comunidades cercanas a las escuelas, de donde provienen sus estudiantes, son diversas y presentan diferentes problemáticas relacionadas con la escasez de recursos y el conflicto social. Aunque las problemáticas relacionadas con el conflicto social se presentan en mayor proporción, no afectan de manera significativa el desempeño de los estudiantes, una vez se controla por el nivel socioeconómico, en cambio las relacionadas con la escasez de recursos, sí tienen una correlación negativa y afectan de manera significativa el desempeño en las áreas de conocimiento evaluadas en el estudio sobre el contexto escolar y social del aprendizaje en Colombia, programa de investigación desarrollado en el marco de las pruebas Saber $3^{\circ}, 5^{\circ}$ y $9^{\circ}$.

La escuela no puede desconocer los escenarios complejos de muchos estudiantes del país, quienes se encuentran en condiciones de vulnerabilidad por las realidades sociales, económicas y culturales de sus familias. Estas situaciones son más evidentes en los habitantes del sector rural, al tener menos posibilidades de acceso a las diversas fuentes del conocimiento y la cultura, según el Icfes (2017), el puntaje promedio obtenido en las pruebas saber por los establecimientos oficiales urbanos en los años 2013, 2014 y 2015, en las áreas de lenguaje y matemáticas es considerablemente mayor al presentado en los establecimientos oficiales rurales en todos los años. Por tal razón, los procesos educativos requieren de docentes ágiles, con la capacidad de indagar acerca de los contextos particulares de sus estudiantes 
y articular esa información en los momentos de planeación, desarrollo y valoración de su práctica pedagógica, si queremos ver a "Colombia como la más educada", eslogan divulgado con frecuencia en los últimos tiempos por el gobierno nacional, pero lejos de alcanzar si las políticas públicas omiten las realidades educativas de cada región y desvaloran la labor del maestro como agente de cambio.

\section{Análisis del contexto y transformaciones en las prácticas educativas}

El contexto interfiere notablemente en las problemáticas de la educación nacional, realidad manifestada en situaciones graves como bajo rendimiento en las evaluaciones censales e internacionales, deserción y repitencia escolar, prácticas pedagógicas ineficientes, entre otras. Como respuesta a esto, se puede iniciar institucionalmente, por analizar el contexto, teniendo en cuenta las perspectivas cultural, antropológica, psicológica y pedagógica, propuestas por Peralta (2002): Desde la perspectiva cultural vale la pena resaltar como la escuela se debe situar en el contexto de la globalización, los intereses nacionales y regionales para planear, ejecutar y retroalimentar el currículo, a fin de responder eficientemente a las necesidades del entorno, del país y del mundo. En relación con la perspectiva antropológica, invita a reflexionar sobre el hecho de ver las aulas como micro sociedades, donde convergen niños, niñas y jóvenes de diversos lugares, con costumbres distintas, tipos de familia diferentes y con historias de vida muy propias, las cuales se deben conocer, con la intención de evitar cualquier tipo de discriminación por las condiciones socioeconómicas o culturales propias de los educandos. En cuanto a la perspectiva psicológica, se deben explorar las posibilidades del aprendizaje de los niños y niñas, teniendo en cuenta las dimensiones cognitiva, afectiva, ética, estética y corporal. La perspectiva pedagógica, hace referencia a saber aprovechar los medios y recursos disponibles, para generar mejores aprendizajes en los estudiantes, utilizando las oportunidades del contexto, como sus desventajas, no para justificar, las problemáticas existentes sino para definir estrategias que permitan construir aprendizajes significativos, desde las realidades de cada grupo poblacional.

Varios autores coinciden en destacar la importancia del contexto en el desarrollo educativo y social, esto es un llamado a repensar las maneras de contribuir en el progreso de los niños y de sus territorios y el posible aporte a la reconfiguración de las mismas políticas públicas, tendientes a garantizar el derecho de los niños y niñas de acceder a una educación de calidad. Según Novoa (2014), “... pensar la sociedad a partir de lo educativo y lo educativo desde la sociedad, forma parte del sistema social y cultural en el que está inmersa; no se educa en lo abstracto sino desde lo cotidiano y concreto" (p.14). En esta misma dirección, Cobo (2008, citado por Novoa, 2014), sostiene que "El compromiso que la educación tiene frente al contexto consiste en preparar a las personas como seres capaces de desarrollar plenamente una ciudadanía democrática ..." (p. 14).

De otra parte, en relación con la realidad circundante, Orozco (2014), indica la necesidad de integrar a todo el corpus social en la solución de los problemas del entorno a partir de observar lo cercano, además comenta como las políticas educativas tanto en Colombia como en otros países, han sido "desinteligentes", pues no han sabido entender los contextos sociales y sus respectivos sueños de nación.

Sin lugar a duda, una de las funciones importantes dadas a la escuela tiene que ver con el asunto académico y el desarrollo como persona, al buscar la formación integral del ser humano para que aporte al progreso social y económico del país. En 
esta perspectiva, para Isaza (2012), el interés común de la familia y la escuela es el desarrollo de los estudiantes. Además señala que si bien en la escuela el aprendizaje pareciera ser el asunto más importante, mientras en la familia es el afecto y la socialización, ambos están encaminados al proceso del crecimiento de la persona. En relación con el aprendizaje y en el caso particular de los modos de producción del conocimiento, Acosta y Carreño (2010), reflexionan sobre como "La producción de conocimiento se aleja cada vez más de la actividad disciplinar para trasladarse a los nuevos contextos sociales" (p. 73). En el caso particular del modo 3, "privilegia la producción de conocimiento altamente especializado en contextos de aplicación orientados a solucionar los problemas de la humanidad y empoderar a las comunidades.". Es necesaria la verdadera transposición didáctica del conocimiento al partir de situaciones reales de los estudiantes y que, al interactuar con la ciencia, se comprenda esas realidades y se aporte a las transformaciones de estas problemáticas.

Respecto al territorio como factor de desarrollo, para Álvarez y Rendón (2010), "la localidad cobra toda la relevancia posible y se convierte en el espacio donde se materializan la acción competitiva y la práctica social, política, económica e institucional que hacen del territorio el eje central del desarrollo y crecimiento económico" (p.47). El territorio, con toda su complejidad incide indudablemente en los procesos formativos desarrollados en la escuela, en consecuencia este debe ser tenido en cuenta en la construcción de los Proyectos Educativos Institucionales y en los mismos planes y programas del Estado. En relación con las políticas públicas, Ibáñez (2013) afirma:

El gobernar por políticas públicas deja de lado la homogenización de las demandas sociales y concibe que los problemas públicos tienen su propia especificidad y contextos por lo cual es necesario que las políticas públicas de intervención también sean específicas y no homogéneas. (p. 40)

Según el Icfes (2014), la política educativa tiene realmente un escenario de acción muy amplio y puede tener un papel activo en la reducción de las diferencias en el logro escolar. Por tal razón, se debe procurar mejores dotaciones de recursos educativos, promoción de climas escolares más apropiado en el desarrollo de las actividades de enseñanza y aprendizaje, mayor involucramiento de los padres de familia en la vida escolar y la detección de factores de riesgo en los estudiantes. $\mathrm{Al}$ abordar estas perspectivas, nos permiten caracterizar mejor nuestros contextos de trabajo, para articularlos a los diseños de clase y prácticas educativas. El reto sigue siendo gigante, ¿cómo transformar los contextos para convertirlos en espacios motivantes o generadores de aprendizajes significativos? ¿Está la escuela preparada para asumir estos desafíos? ¿Cómo lograrlo?... son algunos interrogantes desde los cuales es imperioso empezar a darles otras miradas a las realidades que hacen de la escuela un espacio poco atractivo para nuestros estudiantes y no muy efectivo en el abordaje de las necesidades y exigencias del mundo actual.

En este sentido, las prácticas de enseñanza tienen relación con la afectación sociocultural del acto educativo.

Estas prácticas se refieren a la interacción entre profesor y estudiantes, la cual puede ser positiva si el docente cuenta con un conjunto de habilidades, conocimientos y recursos que permitan desarrollar el aprendizaje de todos los estudiantes en el aula. (Icfes, 2016, p. 33)

Por tal razón, el maestro debe conocer el contexto sociocultural de los estudiantes, las condiciones económicas, las situaciones de convivencia 
en el hogar, el nivel educativo de su entorno familiar... y articular estos hallazgos en sus preparadores de clase, de tal manera que sus prácticas sean incluyentes, valoren la diferencia y propicien ambientes para el logro de aprendizajes y comprensiones profundas.

Por último, para lograr las transformaciones necesarias en la educación actual, se requiere del empeño de todos, estudiantes, padres de familia, profesores, directivos docentes, gobierno nacional y sociedad en general. En esta perspectiva, De Zubiría (2011), señala: "El estado y la sociedad civil se deberán comprometer a establecer la educación como una prioridad nacional, como una verdadera 'locomotora' de la sociedad y del desarrollo humano" (p. 79). De acuerdo con Lobo y Santos (2010), la educación está orientada hacia el desarrollo integral y armónico de la persona y por ello, debe abarcar todos los aspectos y las etapas de la vida del ser humano. Si la educación tiene como único objetivo el desarrollo puramente intelectual y olvida a la persona como unidad cuerpo-menteespíritu, que se debe alimentar en forma integral, difícilmente se logran los propósitos formativos.

\section{Conclusión}

Existe la necesidad de estudiar los contextos circundantes a los procesos de aprendizaje de los estudiantes, con la intención de integrarlos a las prácticas pedagógicas, a fin de convertirlos en una oportunidad para potenciar el desarrollo de competencias académicas, ciudadanas y laborales de los niños, niñas y jóvenes. En este sentido, se propone que próximos investigadores, maestros e instituciones educativas profundicen en las condiciones culturales, sociales y económicas de los estudiantes y familias, mediante instrumentos como cartografía social, entrevistas en profundidad y demás técnicas cualitativas que permitan sistematizar los factores asociados que afectan el rendimiento académico de los estudiantes, con la intención de articular estos hallazgos a la planeación y ejecución de las prácticas de aula.

Comprender las realidades del estudiante permite revisar las prácticas del maestro e integrar los intereses y necesidades; de esta manera el rol de maestro investigador encuentra su razón de ser al poder impactar las maneras de concebir la existencia, el estudio, el trabajo y demás dimensiones humanas de los estudiantes que llegan a la escuela, con el anhelo de aprender. Sin embargo, las mismas dinámicas de los centros educativos desvían sus intereses y terminan muchos ampliando la lista de los repitentes y otros optan por desertar.

De acuerdo a lo anterior, el éxito de la educación en las instituciones educativas, surtirá el efecto deseado si se logra articular a los procesos de enseñanza y de aprendizaje, los diversos contextos socioculturales y las condiciones particulares de los estudiantes. Cuando la escuela consiga concentrar sus esfuerzos en comprender las vivencias de nuestra niñez y juventudes y promover estrategias conforme con sus niveles de desarrollo, tendremos una realidad educativa distinta.

De otra parte, padres de familia como educadores, señalan con preocupación la crisis de la educación, manifestada en los bajos desempeños de los estudiantes en las pruebas internas y externas, en el aumento de la violencia y la proliferación del consumo de drogas en los centros educativos, entre otros aspectos.

Una alternativa ante el panorama descrito anteriormente, es buscar la sinergia entre lo académico y el mundo de la vida de los actores educativos. Los fines de la educación, la realidad de la sociedad y de la población juvenil, parecieran ir por caminos distintos, no se han podido establecer acuerdos entre lo deseado por el Estado y las necesidades reales de sus habitantes. Por ello, se requieren currículos contextualizados, construidos desde el reconocimiento de los territorios, la 
comprensión del entorno sociocultural y del trato diferenciado entre las poblaciones, según las condiciones de vulnerabilidad de cada persona. En definitiva, el contexto sociocultural y el aprendizaje escolar tienen una relación estrecha. El profesor debe procurar armonizar estas dos dimensiones, de tal manera que la primera no sea un obstáculo para desarrollar la segunda.

\section{Referencias}

Acosta, W. y Carreño, C. (2010). Modo 3 de producción de conocimiento: implicaciones para la universidad hoy. Revista de la Universidad de La Salle, 61, pp.67-87.

Álvarez, R. y Rendón, J. (julio-diciembre 2010). El territorio como factor de desarrollo. Medellín: Semestre económico, 13 (27), 39-62.

Argüelles, P. y Nagles, G. (2007). Estrategias para promover procesos de aprendizaje autónomo. Bogotá: Alfaomega colombiana.

Barbero, J M; (1996). Heredando el futuro. Pensar la educación desde la comunicación. Nómadas (Col), () Recuperado de http://www.redalyc.org/ articulo.oa? $\mathrm{id}=105118998002$

Burgos, D. B. y Cifuentes, J. E. (2015). La práctica pedagógica investigativa: entre saberes, quereres y poderes. Revista Horizontes Pedagógicos, 17 (2), 118-127.

Cejudo, R. (2006). Desarrollo humano y capacidades: aplicaciones de la teoría de las capacidades de Amartya Sen a la educación. Revista Española de Pedagogía. Versión digital, 64, (234), 365-382.

De Zubiría, J. (julio-agosto 2011). Un acuerdo nacional para mejorar la calidad de la educación en Colombia, Revista internacional Magisterio, 51, 76-79.

Fernández, C., Fernández, A. y Urrego, K. (2011). Factores socioculturales asociados al aprendizaje de las matemáticas en los estudiantes de la IED San Joaquín de La Mesa Cundinamarca.
(Proyecto de grado para optar el título de Licenciado en Educación Básica con énfasis en Educación Matemática, Universidad del Magdalena, Colombia).

Guzmán, R. y Ghitis, T. (2012). Módulo de contextos de desarrollo y aprendizaje. Maestría en Pedagogía. Universidad de La Sabana.

Icfes. (2010). Saber $5^{\circ}$ y $9^{\circ} 2009$. Resultados Nacionales. Resumen Ejecutivo. Bogotá.

Icfes. (2014). Estudio sobre el contexto escolar y social del aprendizaje en Colombia. Bogotá, octubre 2014. Disponible en: http://www2.icfes.gov. co/seminario/docman/seminario-2014/conferencias-principales-2014/cesac/119-contexto-escolar-y-social-del-aprendizaje-en-colombia-cesaccarolina-lopera/file.html. Recuperado el 5 de diciembre de 2014.

Icfes. (2016). Marco de factores asociados Saber $3^{\circ}$, $5^{\circ}$ y $9^{\circ} 2016$. Bogotá.

Icfes. (2017). Informe nacional. Resultados nacionales 2009, 2012-2015. Saber $3^{\circ}, 5^{\circ}$ y $9^{\circ}$. Bogotá.

Isaza, L. (marzo-abril 2012). La relación familia y escuela y la búsqueda del desarrollo infantil y adolescente, Revista internacional Magisterio, 55, 2227.

Jaramillo, R. (2010). Formación de maestros y contexto social. Medellín: Litoimpresos y servicios Ltda.

Lobo, A. y Santos, R. (2010). Psicología del aprendizaje. Bogotá: Universidad Santo Tomás.

López, N. y Tedesco, C. (2002). Las condiciones de educabilidad de los niños y adolescentes en América Latina Documento para discusión - versión preliminar. Buenos Aires: Instituto Internacional de Planeamiento de la Educación. Disponible en: http://udelas.ac.pa/biblioteca/librospdf/educabilidad.PDF Recuperado el 05 de agosto de 2017.

Martín, J. (s.f.). El caballo estaba dentro. Disponible en: http://www.motivaciones.org/MOTIV003/ ctoseelcaballoestabaadentro.htm Recuperado el 20 de diciembre de 2014.

Ministerio de Educación Nacional (2015). Docente de 
aula. Evaluación de Carácter Diagnóstico Formativa (ECDF). Bogotá.

Ministerio de Educación Nacional (2012). Evaluación de competencias para el ascenso o reubicación de nivel salarial en el escalafón docente de los docentes $y$ directivos docentes regidos por el decreto Ley 1278 de 2012. Documento Guía. Bogotá.

Ministerio de Educación Nacional (1998). Lineamientos Curriculares de Matemáticas. Bogotá.

Novoa, A. (2014). Educación, género y sociedad. En: Cuadernos de seminario 1. Sociedad y Educación: una mirada actual. (13-25). Bogotá: Universidad de La Salle.

Orozco, J. (2014). ¿Para qué sociedad educamos? En: Cuadernos de seminario 1. Sociedad y Educación: una mirada actual. (91-100). Bogotá: Uni- versidad de La Salle.

Peralta, M. (2002). Una pedagogía de las oportunidades. Nuevas ventanas para los alumnos párvulos latinoamericanos del siglo XXI. Andrés Bello. Santiago de Chile: Andrés Bello.

Torres, M. (1995). Repetición escolar: ¿Falla del alumno o falla del sistema? Evaluación, Aportes para la capacitación, 1 . Versión digital. Disponible en: http://www.consultasrodac.sep.gob.mx/cartilla/ pdf/torres_1995.pdf Recuperado el 5 de diciembre de 2014.

Zambrano, A. (2016). Pedagogía y didáctica: esbozo de las diferencias, tensiones y relaciones de dos campos. Praxis \& Saber, 7(13), 45-61.

Zuluaga, O. (2007). Otra vez Comenio. Revista Educación y Pedagogía, 47 (19), 99-118. 